\title{
Xantomatosis cerebrotendínea: U na causa de aumento de volumen bilateral del tendón de Aquiles. Caso clínico
}

\author{
Jorge Filippi $\mathbf{N}^{1}$, Sebastián Irarrázaval $\mathrm{D}^{1}$, Pilar Peredo $0^{2}$, \\ Patricio Mellado $\mathrm{T}^{3}$. \\ Cerebrotendinous xanthomatosis. \\ Report of one case
}

Cerebrotendinous xanthomatosis is an inherited autosomal recessive disease caused by a mutation in the gene for the sterol 27-hydroxylase enzyme, which determines the accumulation of plasmatic cholestanol in various tissues. The natural history of this disease is characterized by chronic diarrhea beginning in childhood, cataract in youth, tendinous xanthomas in adulthood and later progressive neurological dysfunction manifested as dementia, psychiatric disorders, cerebellar, pyramidal or extrapyramidal signs or seizures. We report a 39 year-old male with a history of diarrhea during childhood and bilateral cataracts requiring surgery at 20 years of age, who evolves later with psychiatric disorders and bilateral increased volume in Achilles tendons. High levels of plasmatic cholestanol and magnetic resonance imaging confirmed the diagnosis of this disease (Rev Méd Chile 2009; 137: 815-20).

(Key words: Xanthomatosis, cerebrotendinous; Cholestanol; Achilles tendon)

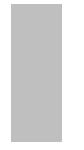

Recibido el 5 de marzo, 2008. Aceptado el 4 de marzo, 2009.

${ }^{1}$ Departamento de Ortopedia y Traumatología, Pontificia Universidad Católica de Chile. Santiago, Chile. ${ }^{2}$ Departamento de Neurología, Universidad de Valparaíso, Chile. ${ }^{3}$ Departamento de Neurología, Pontificia Universidad Católica de Chile. Santiago, Chile.

$\mathrm{L}$ a xantomatosis cerebrotendínea (XCT) es una enfermedad hereditaria autosómica recesiva del metabolismo lipídico. Su prevalencia estimada es de 1 en 50.000 para población caucásica y está causada por una mutación en el gen CYP27A1 (esterol 27-hidroxilasa) del cromosoma $2^{1,2}$. Esta enzima es requerida para la oxidación del coleste-

Correspondencia a: Dr. Jorge Filippi N. Marcoleta $352,1^{\text {er }}$ piso. Teléfono: 3546846. Fax: 3546847. E mail: jfilippi@med.puc.cl rol a ácidos biliares. Su deficiencia produce la acumulación de colestanol plasmático y su depósito en diversos tejidos: cristalino, tendones y sistema nervioso central, lo que determina sus manifestaciones clínicas.

Esta enfermedad puede presentarse inicialmente con aumento de volumen bilateral de los tendones aquilianos ${ }^{3}$. El diagnóstico precoz es fundamental ya que existe tratamiento médico capaz de detener la progresión de la enfermedad y revertir parcialmente sus manifestaciones neurológicas y sistémicas. 


\section{Caso ClínICO}

Paciente hombre de 39 años, hijo único, con padres sanos, soltero y sin hijos. Presentaba antecedentes de diarrea crónica al año de vida, catarata bilateral operada a los 20 años y enfermedad psiquiátrica desde la adolescencia, catalogados de enfermedad bipolar y esquizofrenia. Había recibido múltiples neurolépticos, carbamazepina y litio. Desde hacía 5 años presentaba deterioro cognitivo progresivo, hacía 6 meses se agregaron inestabilidad de la marcha, incontinencia urinaria y aumento de volumen en ambos tobillos. Debido al aumento de volumen bilateral de la región posterior de ambos tobillos consultó en Traumatología. El inicio del aumento de volumen fue insidioso, sin trauma asociado y su evolución progresiva e indolora.

Al examen físico destacaba un aumento del área de sustentación asociado a espasticidad y clonus de ambas extremidades. Se evidenció también un aumento de volumen fusiforme en la región posterior de ambos tobillos, en relación a los tendones aquilianos, desde su inserción distal hasta la unión músculo-tendínea. A la palpación era indolora y de consistencia firme. La piel en la zona del aumento de volumen estaba sana y el examen articular era normal (Figura 1).
Con el diagnóstico presuntivo de xantomatosis cerebrotendínea se solicitó la evaluación de Neurología.

En el examen neurológico destacaba un paciente pueril y con apraxia ideomotora, el examen de nervios craneanos era normal, presentaba una tetraparesia espástica con aumento de los reflejos osteotendíneos, clonus bilateral de extremidades inferiores y signo de Babinski bilateral. En el examen sensitivo destacaba una severa alteración de la sensibilidad postural y vibratoria. Los signos meníngeos estaban ausentes y el examen cerebeloso mostraba una severa ataxia de la marcha, requería de la ayuda de dos personas para caminar y existía una dismetría leve de 4 extremidades. El fondo de ojo era normal.

Se solicitó una resonancia magnética (RM) de encéfalo y de médula espinal además de la determinación de colestanol plasmático (enviado a la Clínica Mayo en Rochester, Estados Unidos de Norteamérica).

La secuencia de FLAIR de la resonancia magnética de encéfalo mostró una hiperintensidad de los núcleos dentados del cerebelo bilateralmente (Figura 2) además de múltiples lesiones hiperintensas supratentoriales inespecíficas. La RM de médula espinal mostró una extensa lesión hiperintensa de las columnas posteriores (Figura 3).

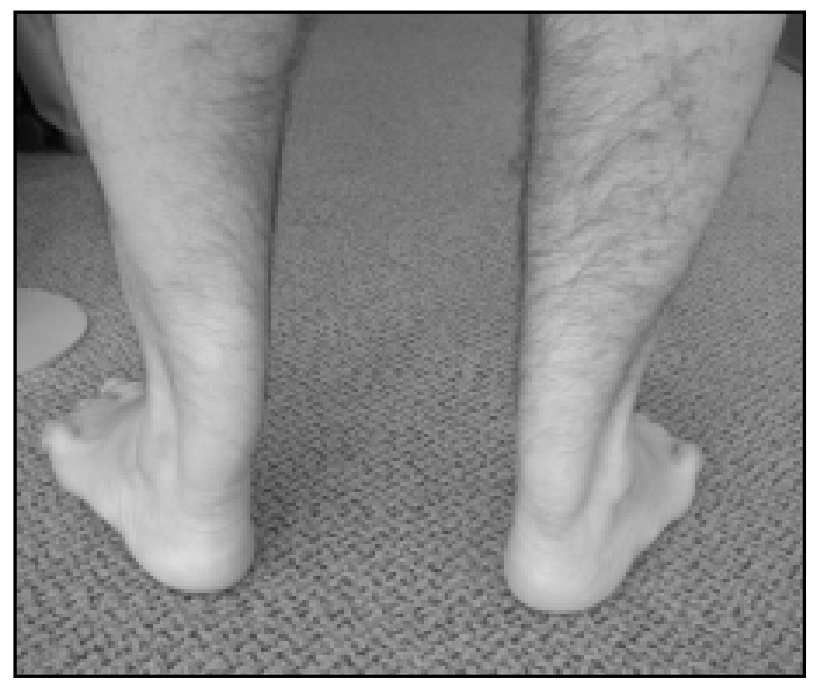

Figura 1. Fotografía que muestra el aumento de volumen bilateral en relación a los tendones aquilianos. 


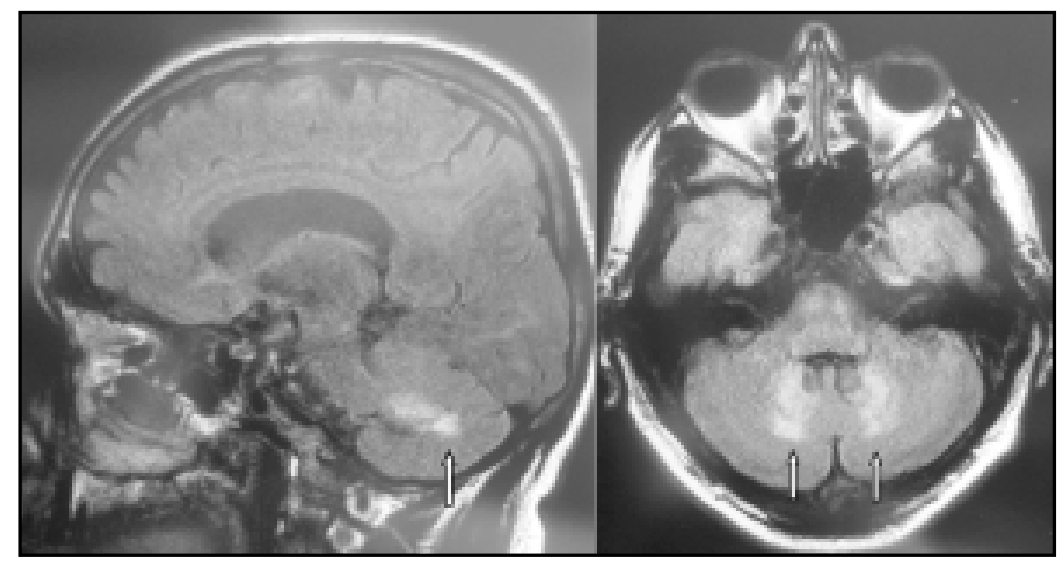

Figura 2. Resonancia magnética de encéfalo en secuencia FLAIR. Lesiones hiperintensas en ambos núcleos dentados (flechas blancas).

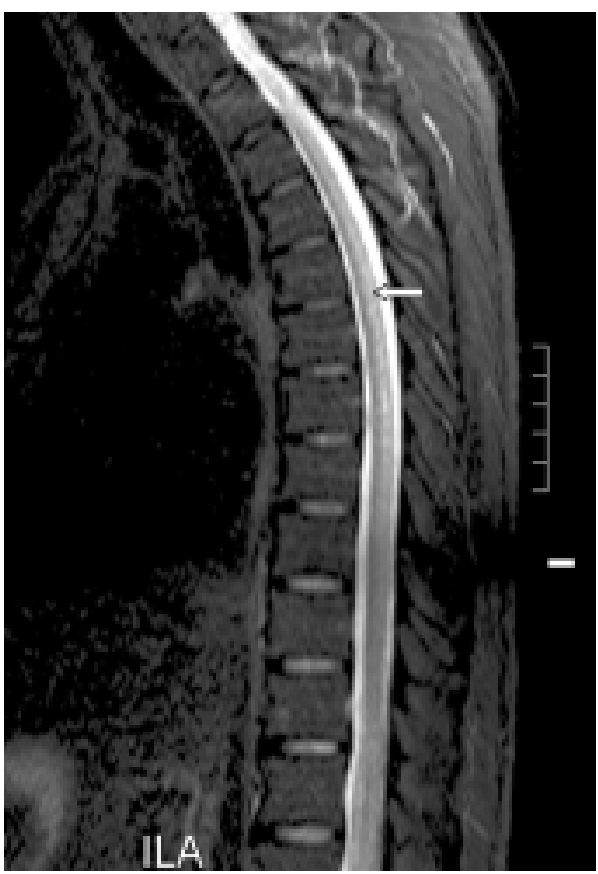

Figura 3. Resonancia magnética de médula espinal mostró hiperintensidad de los cordones posteriores (flecha blanca).

Tanto las lesiones cerebelosas como las de médula espinal eran características de la xantomatosis cerebrotendínea.

El nivel de colestanol fue de $45 \mathrm{mmol} / \mathrm{ml}$ (valor normal menor a $13 \mathrm{Mmol} / \mathrm{ml}$ ), confirman- do el diagnóstico de xantomatosis cerebrotendínea.

Se inició tratamiento con ácido quenodeoxicólico (250 mg cada 8 h). A los 8 meses de tratamiento el paciente presentaba una vida autovalente, logra deambular solo, persiste con incontinencia urinaria y no presentaba problemas psiquiátricos. Los xantomas tendíneos presentaron una disminución de tamaño.

\section{DisCUSIÓN}

La xantomatosis cerebrotendínea fue descrita en 1937 por van Bogaert et $\mathrm{al}^{4}$. La causa es una mutación del gen CYP27A1 que codifica la enzima mitocondrial hepática esterol 27-hidroxilasa, que oxida la cadena lateral del colesterol, paso metabólico necesario para la formación de ácidos biliares.

La deficiencia de esta enzima da lugar a un bloqueo en la síntesis de los ácidos biliares, lo que resulta en una acumulación de productos intermedios como el colestanol y colesterol en los tejidos.

El gen para la esterol 27-hidroxilasa se encuentra en el locus cromosómico 2q33-qter y se han descrito varias mutaciones que pueden producir la enfermedad, sin haber una relación genotipofenotipo demostrada.

La prevalencia publicada de esta condición es mayor en la etnia japonesa y en las comunidades 
judías de África del Norte; sin embargo, la mayoría de los casos son esporádicos ${ }^{5}$. Nuestro paciente no corresponde a ninguno de esos grupos étnicos. Recientemente se reportaron los primeros 2 casos de XCT en Argentina ${ }^{6}$ y hay descritos 2 hermanos brasileños con $\mathrm{XCT}^{7}$. No hay casos chilenos reportados hasta ahora.

La forma de presentación de la enfermedad en este paciente coincide con la historia natural descrita en la literatura. La diarrea crónica se inicia característicamente en la infancia, pudiendo ser la manifestación más precoz ${ }^{8,9}$, sin embargo, hasta en $75 \%$ de los pacientes la manifestación inicial es la aparición de cataratas durante la primera década de la vida ${ }^{10}$, los xantomas tendíneos aparecen en la segunda o tercera década y se pueden observar en el tendón de Aquiles, tendones extensores de codo y mano, patelar y del cuello ${ }^{10}$, la disfunción neurológica puede presentarse a cualquier edad, pero es más clara después de la pubertad, describiéndose en hasta 50\% de los pacientes un deterioro cognitivo progresivo a partir de los 20 años $^{11}$, los signos cerebelosos y piramidales se presentan invariablemente entre los 20 y 30 años $^{12}$. Son frecuentes las alteraciones neuropsiquiátricas, extrapiramidales ${ }^{13}$, convulsiones ${ }^{14}$ y neuropatía periférica, provocando deformidad secundaria o úlceras ${ }^{15-17}$. También se ha descrito osteoporosis ${ }^{18-20}$.

El diagnóstico de la xantomatosis cerebrotendínea puede ser difícil porque los análisis bioquímicos rutinarios de sangre, orina y líquido cefalorraquídeo son a menudo normales.

Las principales alteraciones de laboratorio son la concentración elevada de colestanol plasmático, colesterol plasmático normal o bajo, aumento de los alcoholes biliares urinarios y plasmáticos ${ }^{21,22}$, y aumento de la concentración de colestanol y apolipoproteína B en líquido cefalorraquídeo por cambios en la barrera hematoencefálica. Nuestro paciente presentaba niveles plasmáticos de colestanol aproximadamente 4 veces sobre el valor normal, compatibles con el diagnóstico de xantomatosis cerebrotendínea.

Apoyan al diagnóstico en nuestro paciente los hallazgos en la RM de encéfalo. Característicamente se describe atrofia difusa, hiperintensidad bilateral de los núcleos dentados, globo pálido, sustancia nigra, oliva inferior y sustancia blanca cerebelosa y cerebral, en secuencia T2 y FLAIR. La
RM de médula espinal en secuencia T2 presenta aumento de señal en columnas laterales y dorsa$\operatorname{les}^{23}$. La espectroscopia por RM muestra disminución de n-acetilaspartato y aumento de lactato, indicadores de disfunción mitocondrial ${ }^{24}$.

En un paciente que se presenta con xantomas tendíneos el diagnóstico diferencial incluye patologías genéticas como: 1) Sitosterolemia, producida por depósito de esterol. Cursa con ateromatosis prematura y no se describen catarata ni signos neurológicos primarios ${ }^{25}$. 2) Hipercolesterolemia e hiperlipemia, en las cuales los niveles de colestanol son normales. En los casos de XCT sin xantomas evidentes, el diagnóstico diferencial incluye todas las formas de deterioro neurológico progresivo $^{11}$. La primera causa genética de catarata de inicio precoz es la distrofia miotónica tipo 1 y la segunda la XCT. La asociación de catarata juvenil, diarrea crónica y deterioro mental progresivo, sugieren fuertemente una $\mathrm{XCT}^{11}$. Esta fue la forma de presentación en nuestro paciente.

El estudio diagnóstico puede complementarse con la determinación de la actividad enzimática de la esterol 27-hidroxilasa en fibroblastos o leucocitos, la que está disminuida en individuos afecta$\operatorname{dos}^{26}$. Es posible la confirmación diagnóstica mediante estudio genético molecular con la secuenciación del gen, que permite detectar 90\% a $100 \%$ de las mutaciones responsables de la enfermedad $^{5}$. En nuestro paciente no fue necesario realizar el estudio molecular por lo característico del cuadro clínico y los niveles de colestanol.

El tratamiento de la xantomatosis cerebrotendínea se basa en la normalización de la síntesis de ácidos biliares con ácido quenodeoxicólico, logrando la reducción de los niveles de metabolitos anormales del plasma, bilis y orina. También suprime la biosíntesis de colestanol, normalizándose sus concentraciones en plasma y líquido cefalorraquídeo. Este tratamiento mejora las manifestaciones neurológicas $^{27,28}$, manifestaciones sistémicas como los xantomas $^{29-32}$ e incluso la osteoporosis ${ }^{33}$. Desafortunadamente no hay disponibilidad del medicamento en Chile por lo que en este caso fue necesario importarlo. Los inhibidores de la HMG-CoA reductasa sola o en combinación con ácido quenodeoxicólico también han demostrado ser eficaces en disminuir los niveles de colestanol y mejorar los signos clínicos ${ }^{32,34}$. 
El paciente que presentamos recibió tratamiento con ácido quenodeoxicólico, revirtiendo par-

\section{REFERENCIAS}

1. Lee MH, Hazard S, Carpten JD, Yi S, Cohen J, Gerhardt GT ET AL. Fine-mapping, mutation analyses, and structural mapping of cerebrotendinous xanthomatosis in U.S. pedigrees. J Lipid Res 2001; 42: 159-69.

2. Bjorken I, Boberg KM, Leitersdoef E. Inborn errors in bile acid biosynthesis and storage of sterols other than cholesterol. In: Scriver CR, Beaudet AL, editors. The metabolic and molecular bases of inherited disease. $8^{\text {th }}$ ed. New York: McGraw-Hill; 2001; 2970-8.

3. Brodsky JW, Beischer AD, Anat D, East C, Soltero E, TINT GS ET AL. Cerebrotendinous xanthomatosis: a rare cause of bilateral Achilles tendon swelling and ataxia. A case report. J Bone Joint Surg Am 2006; 88: $1340-4$.

4. van Bogaert L, Scherer HJ, Epstein E. Une forme cérébrale de la cholestérinose généralisée: (type particulier de lipidose à cholestérine). Paris: Masson et Cie; 1937.

5. Meiner V, Meiner Z, Reshef A, Bjorkhem I, Leitersdorf E. Cerebrotendinous xanthomatosis: molecular diagnosis enables presymptomatic detection of a treatable disease. Neurology 1994; 44: 288-90.

6. Szlago M, Gallus GN, Schenone A, Patiño Me, Sfaelo Z, Rufa A ET AL. The first cerebrotendinous xanthomatosis family from Argentina : a new mutation in CYP27A1 gene. Neurology 2008; 70: 402-4.

7. Lange MC, Zétola Vf, Teive HA, Scola RH, Trentin AP, Zavala JA ET aL. Cerebrotendinous xanthomatosis: reporto f two Brazilian Brothers. Arq Neuropsiquiatr 2004; 62: 1085-9.

8. Cruysberg JR, Wevers RA, Tolboom JJ. Juvenile cataract associated with chronic diarrhea in pediatric cerebrotendinous xanthomatosis. Am J Ophthalmol 1991; 112: 606-7.

9. Cruysberg JR. Cerebrotendinous xanthomatosis: juvenile cataract and chronic diarrhea before the onset of neurologic disease. Arch Neurol 2002; 59: 1975.

10. Cruysberg JR, Wevers RA, van Engelen BG, Pinckers A, van Spreeken A, Tolboom JJ. Ocular and systemic manifestations of cerebrotendinous xanthomatosis. Am J Ophthalmol 1995; 120: 597-604.

11. Verrips A, van Engelen BG, Ter Laak H, GabreelsFesten A, Janssen A, Zwarts M et al. Cerebrotendinous xanthomatosis. Controversies about nerve and muscle: observations in ten patients. Neuromuscul Disord 2000; 10: 407-14.

12. Verrips A, Nijeholt GJ, Barkhof F, Van Engelen BG, Wesseling P, Luyten JA et al. Spinal xanthomatosis: a cialmente su sintomatología neurológica y sus xantomas tendíneos.

variant of cerebrotendinous xanthomatosis. Brain 1999; 122: 1589-95.

13. Grandas F, Martin-Moro M, García-Munozguren S, ANAYA F. Early-onset parkinsonism in cerebrotendinous xanthomatosis. Mov Disord 2002; 17: 1396-7.

14. Arlazoroff A, Roitberg B, Werber E, Shidlo R, Berginer VM. Epileptic seizure as a presenting symptom of cerebrotendinous xanthomatosis. Epilepsia 1991; 32: 657-61.

15. Kuritzky A, Berginer VM, Korczyn AD. Peripheral neuropathy in cerebrotendinous xanthomatosis. Neurology 1979; 29: 880-1.

16. Kuriyama M, Fujiyama J, Yoshidome H, Takenaga $S$, Matsumuro K, Kasama T et al. Cerebrotendinous xanthomatosis clinical and biochemical evaluation of eight patients and review of the literature. J Neurol Sci 1991; 102: 225-32.

17. Katz DA, Scheinberg L, Horoupian DS, Salen G. Peripheral neuropathy in cerebrotendinous xanthomatosis. Arch Neurol 1985; 42: 1008-10.

18. Berginer VM, Shany S, Alkalay D, Berginer J, Dekel S, SAlEN G ET al. Osteoporosis and increased bone fractures in cerebrotendinous xanthomatosis. Metabolism 1993; 42: 69-74.

19. Chang WN, Lui CC. Failure in the treatment of longstanding osteoporosis in cerebrotendinous xanthomatosis. J Formos Med Assoc 1997; 96: 225-7.

20. Kuriyama M, Fujiyama J, Kubota R, Nakagawa M, Osame M. Osteoporosis and increased bone fractures in cerebrotendinous xanthomatosis. Metabolism 1993; 42: 1497-8.

21. Bouwes Bavinck JN, Vermeer BJ, Gevers Leuven JA, Koopman BJ, Wolthers BG. Capillary gas chromatography of urine samples in diagnosing cerebrotendinous xanthomatosis. Arch Dermatol 1986; 122 : 1269-72.

22. Wolthers BG, Volmer M, van der Molen J, Koopman BJ, De Jager AE, Waterreus RJ. Diagnosis of cerebrotendinous xanthomatosis (CTX) and effect of chenodeoxycholic acid therapy by analysis of urine using capillary gas chromatography. Clin Chim Acta 1983; 131: 53-65.

23. Barkhof F, Verrips A, Wesseling P, van der KnaAp M, van Engelen B, Gabrë̈ls $F$ et al. Cerebrotendinous Xanthomatosis: The Spectrum of Imaging Findings and the Correlation with Neuropathologic Findings. Radiology 2000; 217: 869-76.

24. De Stefano N, Dotti MT, Mortilla M, Federico A. Magnetic resonance imaging and spectroscopic changes in brains of patients with cerebrotendinous xanthomatosis. Brain 2001; 124: 121-31. 
25. Hatanaka I, Yasuda H, Hidaka H, Harada N, Kobayashi M, OKabe H et al. Spinal cord compression with paraplegia in xanthomatosis due to normocholesterolemic sitosterolemia. Ann Neurol 1990; 28: 390-3.

26. Moghadasian MH. Cerebrotendinous xanthomatosis: clinical course, genotypes and metabolic backgrounds. Clin Invest Med 2004; 27: 42-50.

27. Mondelli M, Rossi A, Scarpini C, Dotti MT, Federico A. Evoked potentials in cerebrotendinous xanthomatosis and effect induced by chenodeoxycholic acid. Arch Neurol 1992; 49: 469-75.

28. Mondelli M, Sicurelli F, Scarpini C, Dotti MT, Federico A. Cerebrotendinous xanthomatosis: 11-year treatment with chenodeoxycholic acid in five patients. An electrophysiological study. J Neurol Sci 2001; 190: 29-33.

29. Nakamura T, Matsuzawa Y, Takemura K, Kubo M, Miki $\mathrm{H}$, Tarui S. Combined treatment with chenodeoxycholic acid and pravastatin improves plasma cholestanol levels associated with marked regression of tendon xanthomas in cerebrotendinous xanthomatosis. Metabolism 1991; 40: 741-6.

30 Kuriyama M, Tokimura Y, Fujiyama J, Utatsu Y, Osame M. Treatment of cerebrotendinous xanthomatosis: effects of chenodeoxycholic acid, pravastatin, and combined use. J Neurol Sci 1994; 125: 22-8.

31. Watts GF, Mitchell WD, Bending JJ, Reshef A, LEITERSDORF E. Cerebrotendinous xanthomatosis: a family study of sterol 27-hydroxylase mutations and pharmaco-therapy. QJM 1996; 89: 55-63.

32. Peynet J, Laurent A, De Liege P, Lecoz P, Gambert P, Legrand A ET AL. Cerebrotendinous xanthomatosis: treatments with simvastatin, lovastatin, and chenodeoxycholic acid in 3 siblings. Neurology 1991; 41: 434-6.

33. Federico A, Dotti MT, Lore F, Nuti R. Cerebrotendinous xanthomatosis: pathophysiological study on bone metabolism. J Neurol Sci 1993; 115: 67-70.

34. Verrips A, Wevers RA, Van Engelen BG, Keyser A, Wolthers BG, BARKHOF F ET AL. Effect of simvastatin in addition to chenodeoxycholic acid in patients with cerebrotendinous xanthomatosis. Metabolism 1999; 48: 233-8. 